\title{
Influence of long-term treatment with a green tea (Camellia sinensis) decoction with or without citrus juice on liver steatosis and some plasma variables in the elderly rat
}

\author{
M. Hamdaoui ${ }^{1}$, Z. Ben Abid $^{1}$, J. Akrem ${ }^{1}$ and H. Jaafoura ${ }^{2}$ \\ ${ }^{1}$ Ecole Supérieure des Sciences et Techniques de la Santé, Tunis, Tunisia and ${ }^{2}$ Faculté de Médecine de Tunis, Tunis, Tunisia
}

The aim of the present study was to examine the influence of long-term treatment with a green tea decoction (GTD) with or without citrus juice (CJ; high ascorbic acid content) on liver steatosis and some plasma variables in the elderly rat.

Twenty-eight male Wistar rats were used in the study. After an adjustment period of 1 week, rats were weighed and randomly assigned to four groups of seven animals with comparable body weights. During the experimental period ( 9 weeks) the rats were given a high-fat diet $(30 \%$ animal fat $+1 \%$ cholesterol; HDF) to promote weight gain and liver steatosis. Group 1 received a normal-fat diet (NDF) + distilled water, group 2 received HDF + distilled water (CG2), group 3 received HDF + GTD and group 4 received HDF + a mixture of CJ (as a source of ascorbic acid) and GTD (CJGTD). At the end of the experimental period the rats were weighed and then killed. A fasting blood sample was withdrawn and plasma glucose, TAG and total cholesterol were determined. $\mathrm{Fe}, \mathrm{Cu}$ and $\mathrm{Zn}$ status was also determined in whole blood and in the liver. Histological examination was carried out after treatment using light microscopy. The results were analysed by ANOVA.

GTD did not influence the daily food intake among groups (mean 13.7 (SE 0.2 ) g/d). On the other hand, the initial body weights did not differ among groups (mean 216 (SE 6) g). However, at the end of the experimental period rats that consumed GTD had lower weight gains than the control group (CG2; $P<0.001)$; weight gains were $46,17.6$ and $26.1 \%$ for the CG2, GTD and CJGTD groups respectively. Nevertheless, GTD did not significantly influence the relative liver weights. Plasma glucose and TAG levels were significantly decreased by 100 and $35 \%$ respectively only in the GTD group. Histological analysis of the liver showed a significant regression of steatosis in the GTD group compared with the CG2 and CJGTD groups. Moreover, the hepatocytes contained considerably more steatosis-related microand macrovacuoles in the CG2 and CJGTD groups than in the GTD group. In the GTD group the hepatocyte cytoplasm contained a significantly lower number of steatosis-related vacuoles in some hepatic cells than the other treatment groups. However, steatosis-related vacuoles were not visible in the NDF group.

GTD significantly reduces all plasma lipid variables and non-alcohol steatosis-related micro- and macrovacuoles in elderly rats fed HDF. 